LA-UR- Ma Approved for public release
distribution is unlimited.

c.l

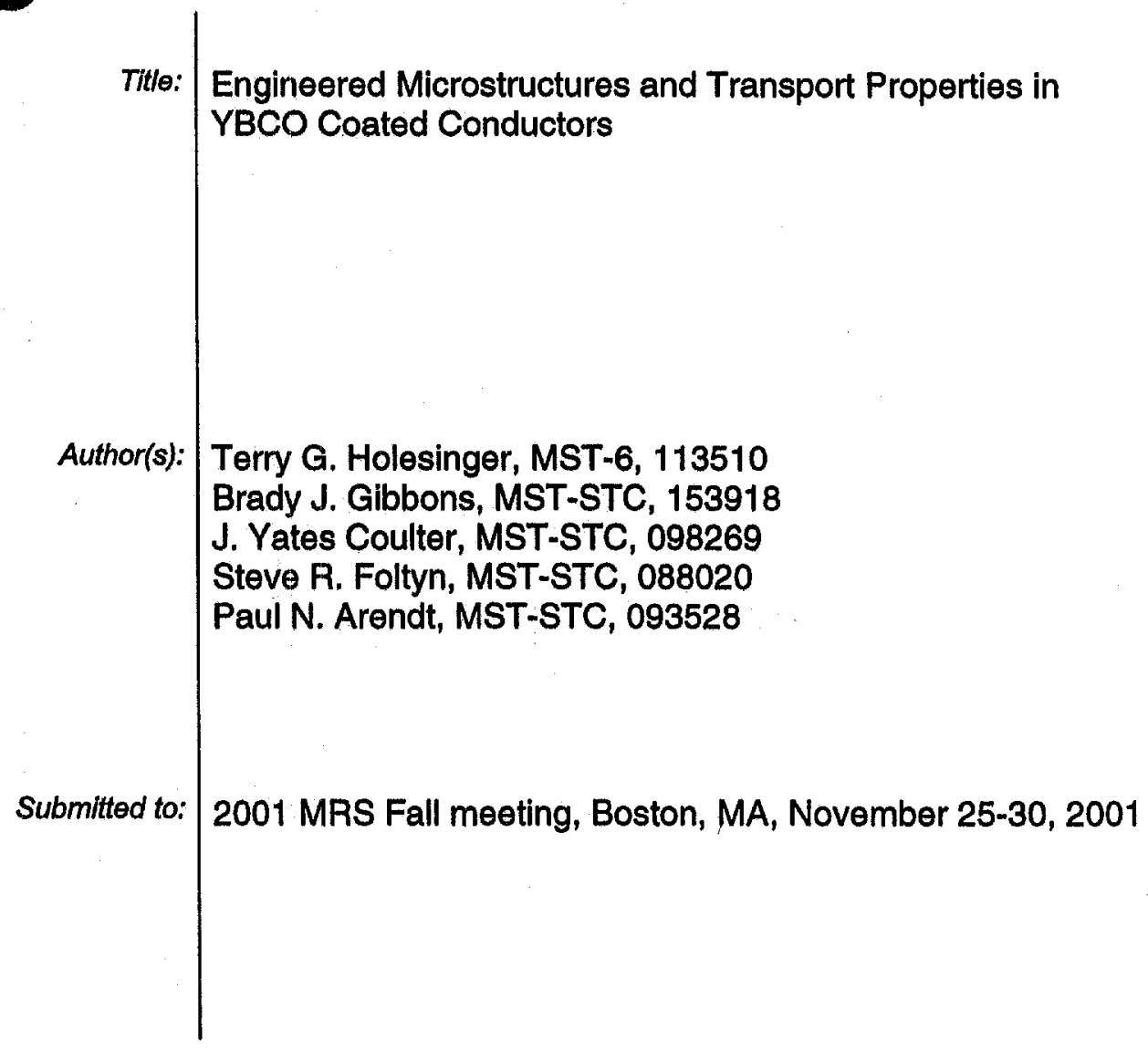

Los Alamos National Laboratory, an affirnative action/equal opportunily employer, is operated by the University of Califomia for the U.S. Department of Energy under contract W-7405-ENG-36. By acceptance of this article, the p $\equiv$ ier recognizes that the U.S. Government retains a nonexclusive, royalty-free license to publlsh or reproduce the published form of th $=$ itribution, or to allow others to do so, for U.S. Govemment purposes. Los Alamos National Laboratory requests that the publisher identify uns article as work performed under the ausplces of the U.S. Department of Energy. Los Alamos National Laboratory strongly supports academlc freedom and a researcher's right to publish; as an institution, however, the Laboratory does not endorse the vlewpoint of a publication or guarantee its technical correctness. 


\title{
Engineered Microstructures and Transport Properties in YBCO Coated Conductors
}

\author{
T.G. Holesinger, B.J. Gibbons, J.Y. Coulter, S.R. Foltyn, J.R. Groves, and P.N. Arendt \\ Materials Science and Technology Division, Los Alamos National Laboratory, \\ Los Alamos, NM 87545 U.S.A.
}

\begin{abstract}
Each process used to deposit or make the bi-axially textured template, buffer layer(s), and the superconductor in a coated conductor creates interfaces along which defects or interfacial reactions may result. These defects can be additive and propagate through the entire film structure to affect the growth and properties of the superconducting film. Defects within the films and their corresponding transport properties have been correlated with the differences in the thickness of the underlying buffer layer material. This knowledge can be used to control and engineer the structure of the coated conductor to maximize critical current densities.
\end{abstract}

\section{INTRODUCTION}

The production of the so-called second-generation "coated conductors" requires a biaxially-textured template for the subsequent oriented growth of additional oxide buffer layers and $\mathrm{YBa}_{2} \mathrm{Cu}_{3} \mathrm{O}_{\mathrm{y}}(\mathrm{YBCO})$ [1-3]. Ion-beam-assisted deposition (IBAD) is one approach that is used for preparing a biaxially-aligned buffer layer on polycrystalline nickel-alloy substrates. In general, YBCO films may be deposited directly onto the IBAD YSZ or MgO templates. However, additional buffer layers have been found to be beneficial in optimizing the transport properties and controlling defects in the Y-123 films [4-9].

Because the coated conductor process is a layering process, several interfaces are formed along which defects or interfacial reactions may result. These defects can be additive and propagate through the entire film structure to affect the growth and properties of the YBCO film. Structural defects, chemical defects, or a combination of both are often formed along the various interfaces $[6,7,10-12]$. Their origins include substrate roughness, lattice mismatch, porosity, contamination, adhesion problems, and interfacial reactions.

One area of specific concern is the underlying buffer layer to the $\mathrm{YBCO}$ film. $\mathrm{CeO}_{2}$ is often used successfully as this buffer layer as it is a stable oxide with a good lattice match to YBCO $[3,4,13]$. However, even ceria reacts with YBCO and affects the superconductor's properties [6]. Since it appears that nearly all materials that have been used as buffer layers react with Y-123 at typical processing temperatures, it is especially important to understand what general aspects of the interfacial reactions between a given material and Y-123 have on the superconducting transport properties. In this paper, various aspects of defect generation in YBCO coated conductors are discussed. Particular attention was paid to the role of the underlying buffer layer to the YBCO and how its thickness can influence the stability of the interface and the properties of the superconducting film.

\section{EXPERIMENTAL}

IBAD was used to produce the biaxially-textured YSZ films on Inconel 625 or Hastelloy substrates. The IBAD YSZ thickness was kept at $0.5 \mu \mathrm{m}$. Ceria and Y-123 layers were 

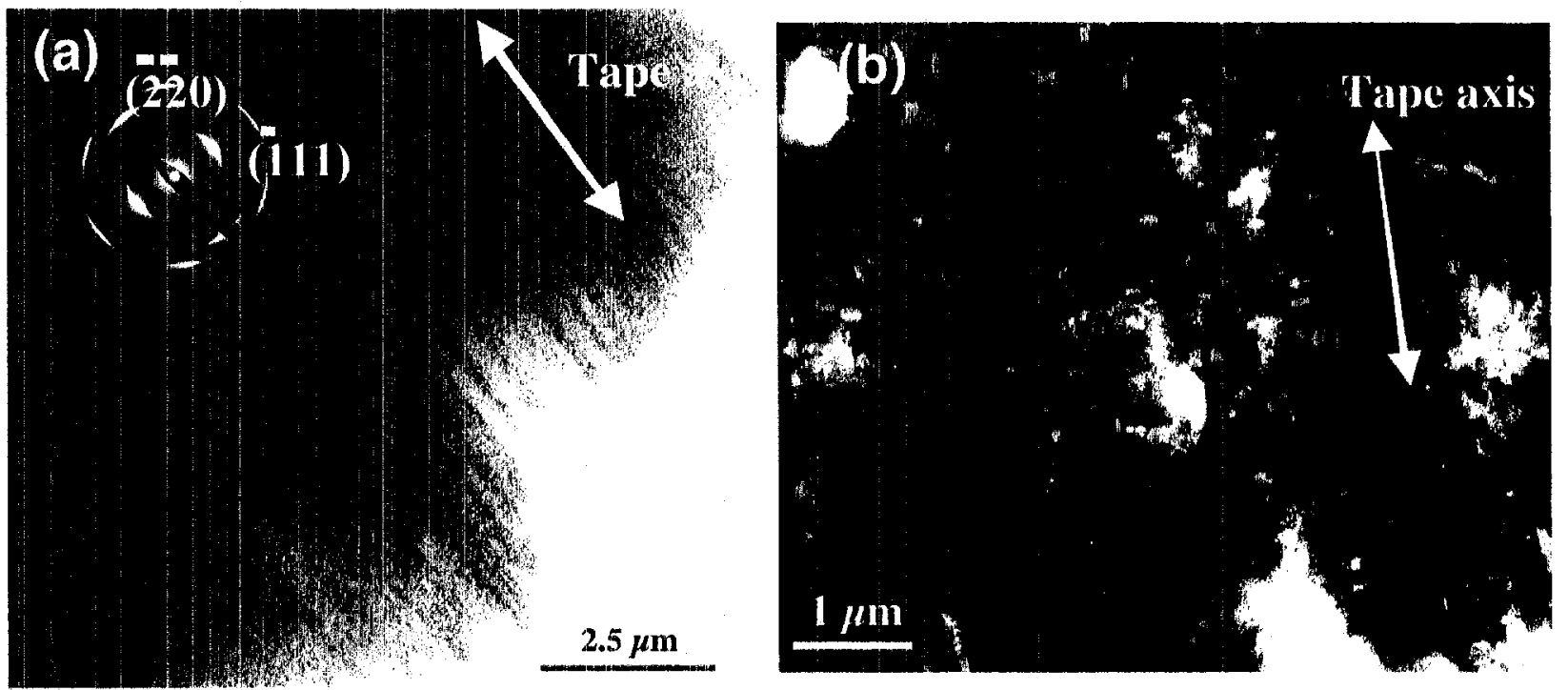

Figure 1: Plan view TEM micrographs of (a) the IBAD YSZ layer and (b) the YBCO layer of a coated conductor. The images were taken with the beam nominally along the [1-12] and [001] zone axes, respectively. Local misorientations in the YSZ or YBCO from these conditions cause the material to appear brighter in the micrographs. The striped nature of the contrast along the tape axis arises from slight deviations in the alignment of the material due to the surface morphology of the substrate. The small, bright rectangular grains in (b) are a-axis oriented grains.

deposited by pulsed laser deposition (PLD) on either the IBAD YSZ templates or single-crystal YSZ substrates. The thickness of the ceria and Y-123 layers was varied between 5 and $1000 \mathrm{~nm}$ and 1 and 3 microns, respectively. Additional details of the deposition processes can be found elsewhere $[5,14,15]$.

Transmission and scanning electron microscopy (TEM and SEM) samples were prepared in the same manner for viewing in the longitudinal transverse direction (perpendicular to both the nominal c-axis of the film and direction of current flow) [6]. Dimple polishing and ion-milling with $3.4 \mathrm{kV}$ Ar ions were used for TEM sample preparation. Phase identification was performed by $\mathrm{x}$-ray diffraction, energy dispersive spectroscopy (EDS) and electron diffraction.

\section{DISCUSSION}

\section{Effects of Substrate Defects}

The growth of the Y-123 film has been observed to change in order to accommodate substrate and buffer layer defects. Substrate defects that can affect YBCO film growth include surface roughness, cracks, pits or holes, bubbles, and substrate inclusions. Their effects may be reflected in the local alignment of the YBCO film, a structural defect in the YBCO film, the occurrence of an interfacial reaction phase, or a combination thereof. The TEM micrographs of Figure 1 dernonstrate how irregularities in the surface morphology of the substrate can affect film alignment. Rolling and polishing marks can affect the local out-of-plane alignment of the films perpendicular to the long axis of the tapes resulting in the striped contrast present in the 

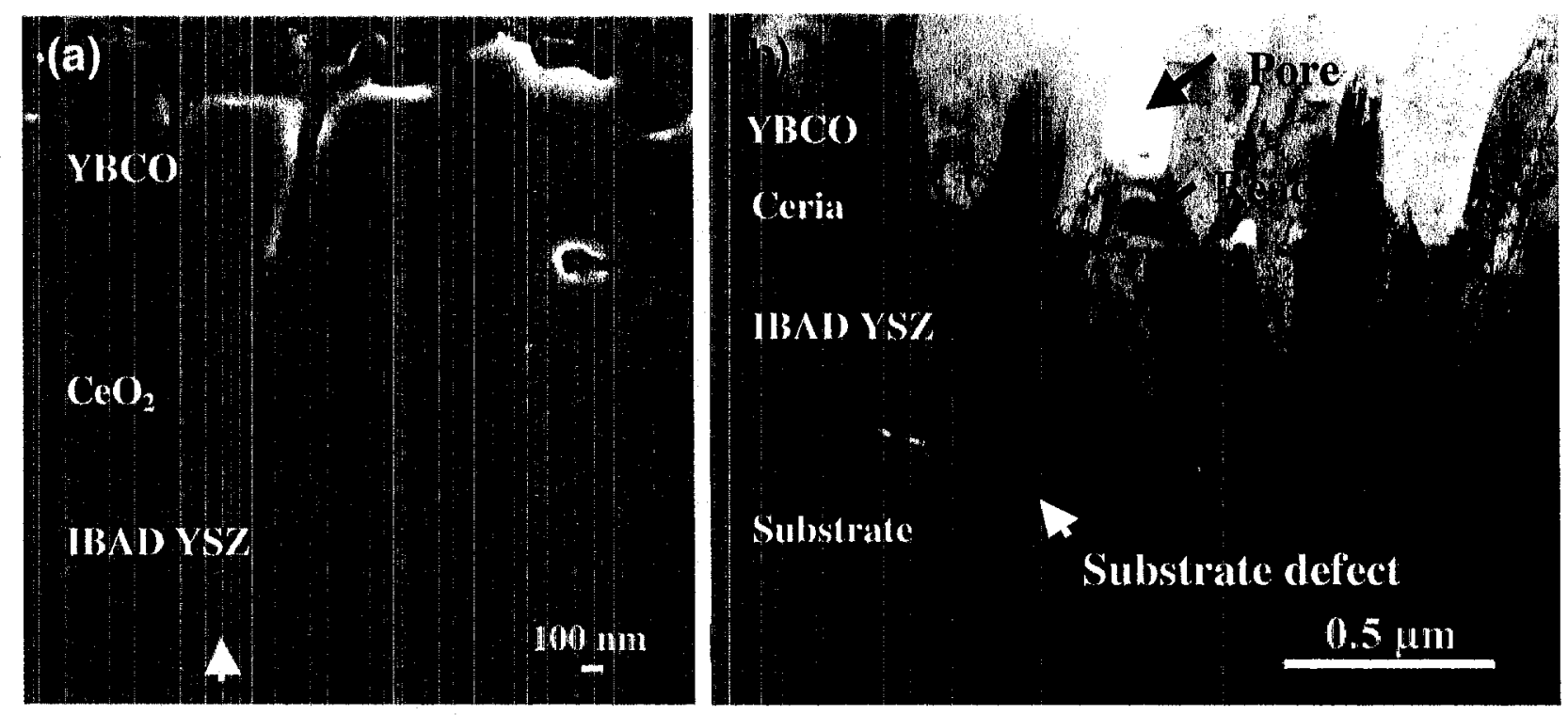

Figure 2: Examples of localized substrate defects and their affects on the YBCO film growth. The SEM micrograph in (a) shows the formation of a large pore structure in the YBCO film that can be traced to a substrate defect. The TEM micrograph (b) shows the sequence of events that starts with the substrate defect and ends with the formation of a large pore structure in the YBCO film.

TEM micrographs. Localized defects in the substrates can also propagate through the buffer layers and increase the likelihood of an interfacial reaction between the YBCO film and the underlying buffer layer. Shown in Figure 2 are SEM and TEM micrographs of substrate defects that have propagated through the intervening buffer layers. The SEM micrograph of Figure 2a shows a large pore strusture in the YBCO film that is associated with a pit in the substrate. The TEM micrograph in Figure $2 \mathrm{~b}$ reveals a sequence of events in which a localized defect in the substrate affected the local alignment of the IBAD YSZ and ceria films. This was then followed by a severe interfacial reaction between the ceria and YBCO. Above this reaction zone, a large pore structure can be seen in the YBCO film. The $J_{c}$ and thickness of the film were $1.7 \mathrm{MA} / \mathrm{cm}^{2}$ (75K, SF) and $1.5 \mu \mathrm{m}$, respectively. The source of almost all the observed interfacial reactions between the YBCO and ceria in this sample could be traced to substrate defects.

No interfacial ractions were observed between the IBAD YSZ and ceria layers. However, the morphology of the interface will follow that of the underlying substrate and, as shown above, defects fiom the substrate can propagate through this interface and affect the films above it. However, even in the absence of the substrate defects, the ceria layer will in general react with the YBCO film. Shown in Figure 3 are TEM micrographs of the types of reactions one finds along the interface. In some cases, the YBCO can maintain its alignment as shown in Figure $3 \mathrm{a}$. In other cases, the reaction phases can cause the growth of misoriented YBCO grains as shown in Figure $3 \mathrm{~b}$. The overall chemistry of the reactions has been documented in a previous paper [7]. The reaction always results in the formation of $\mathrm{BaCeO}_{3}$. When the reaction is severe, $\mathrm{YCuO}_{2}$ is formed above the $\mathrm{BaCeO}_{3}$ in these films. When the reaction is small, leftover material from the formation of $\mathrm{BaCeO}_{3}$ can diffuse along the grain boundary and intercalate as intergrowths of $\mathrm{Y}_{2} \mathrm{Ba}_{4} \mathrm{Cu}_{7} \mathrm{O}_{y}(\mathrm{Y}-247)$ or $\mathrm{Y}_{2} \mathrm{Ba}_{4} \mathrm{Cu}_{8} \mathrm{O}_{\mathrm{y}}(\mathrm{Y}-248)$. An example of the 

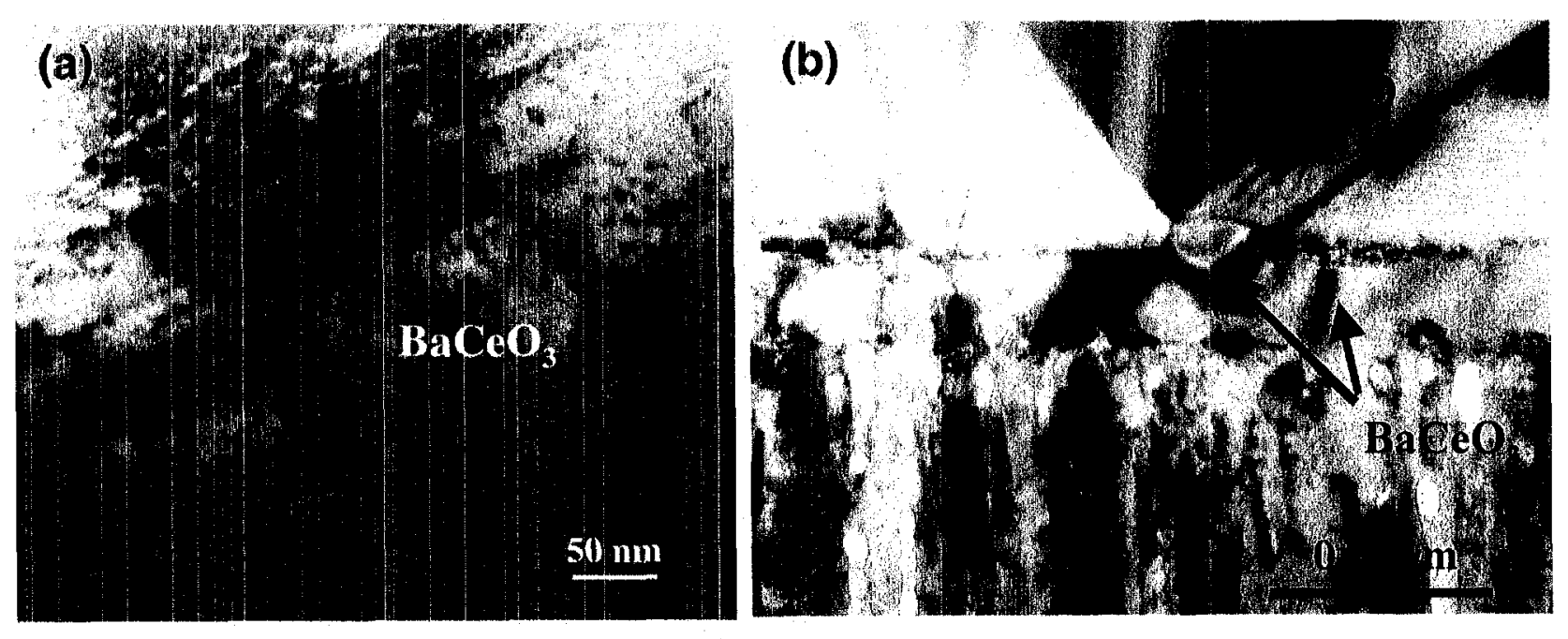

Figure 3: TEM micrographs of examples in which (a) the alignment of the YBCO was not affected by the formation of $\mathrm{BaCeO}_{3}$ during the interfacial reaction and (b) a [103] oriented YBCO grain that nucleated on a $\mathrm{BaCeO}_{3}$ grain at the interface.

latter is shown in Figure 4. The line in Figure 4 indicates where a discontinuity in the ceria layer is associated with an interfacial reaction that formed the $\mathrm{BaCeO}_{3}$.

\section{$\mathrm{CeO}_{2}$ Thickness Effects}

It is clear from the above discussion that eliminating substrate defects will reduce the

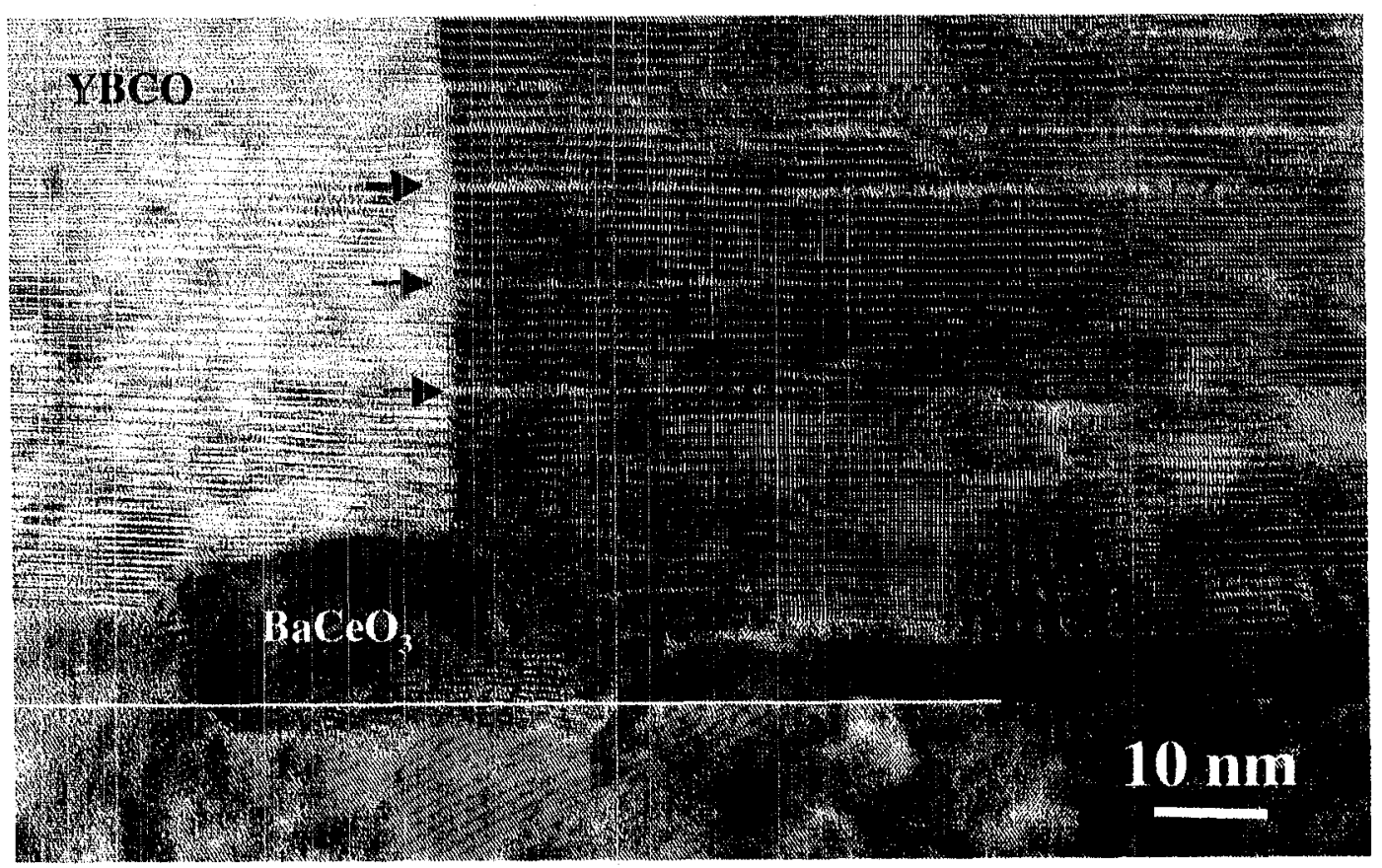

Figure 4: TEM micrograph of an incomplete interfacial reaction. Material left over from the reaction to form $\mathrm{BaCeO}_{3}$ diffuses along the grain boundary and intercalates as intergrowths of $\mathrm{Y}_{2} \mathrm{Ba}_{4} \mathrm{Cu}_{7} \mathrm{O}_{\mathrm{y}}$ and $\mathrm{Y}_{2} \mathrm{Ba}_{4} \mathrm{Cu}_{8} \mathrm{O}_{\mathrm{y}}$. The white line is a guide to the eye to locate the discontinuity in the ceria layer and the resulting interfacial reaction that formed $\mathrm{BaCeO}_{3}$. 

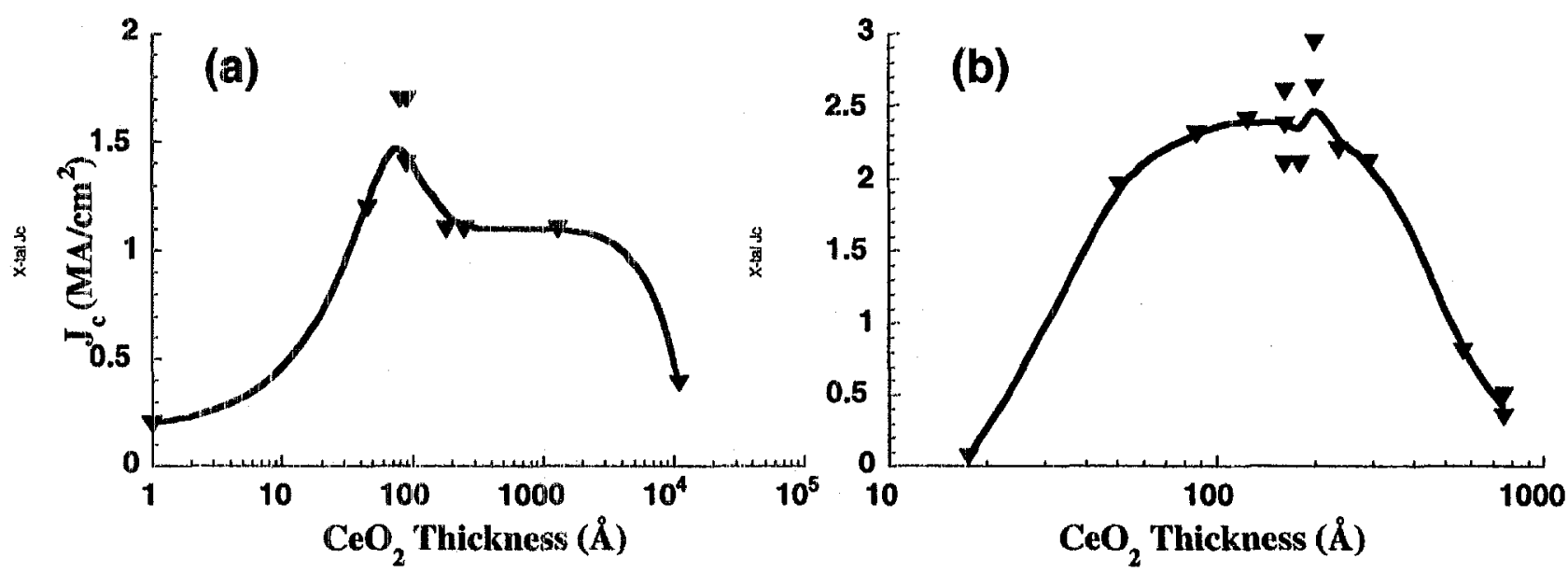

Figure 5: Plot of the $J_{c}$ values as a function of the thickness of the buffer layer beneath the YBCO film for (a) IBAD YSZ templates and (b) single-crystal YSZ substrates. The thickness averages of the YBCO films were 1.5 and $1.0 \mu \mathrm{m}$, respectively.

number of interfacial reactions between the YBCO and underlying buffer layer. A second way to reduce the interfacial reactions is to optimize the thickness of the underlying buffer layer, which in this case is ceria. Shown in Figure $5 \mathrm{a}$ and $\mathrm{b}$ are plots of the critical current density $\left(\mathrm{J}_{\mathrm{c}}\right)$ as a function ceria thickness for Y-123 films deposited on IBAD YSZ-coated metal substrates and YSZ single-crystal substrates. This maximum in $J_{c}$ in both cases correlates with a minimum in the reactivity between the ceria buffer layer and Y-123 film. The critical ceria layer thicknesses on the IBAD YSZ metal tapes and single crystals were approximately $90 \AA$ and 200 $\AA$, respectively. The maximum $J_{c}$ value reached with a $90 \AA$ ceria film on a metal substrate and a $1.5 \mu \mathrm{m} Y-123$ film was $1.7 \mathrm{MA} / \mathrm{cm}^{2}$ at $75 \mathrm{~K}$ and self-field. A maximum $J_{c}$ value of $2.9 \mathrm{MA} / \mathrm{cm}^{2}$ was reached for a $1 \mu \mathrm{m}$ thick YBCO film on a single-crystal YSZ substrate with an intervening $200 \AA$ ceria buffer layer.

At the critical thickness, the interfacial reactions were found to be almost non-existent as shown in the example of a single-crystal substrate in Figure 6 . In contrast, relatively small increases or decreases in the thickness of the ceria buffer layer relative to the optimal thickness induced significant interfacial interactions. Shown in Figure 7 are cross-sectional and plan view TEM images of YBCO films on a single-crystal substrates where the intervening ceria buffer layer of thicknesses were 560 and $800 \AA$, respectively. The corresponding $J_{c}$ values in the $1 \mu \mathrm{m}$ thick films were 0.8 and $0.35 \mathrm{MA} / \mathrm{cm}^{2}$ at $75 \mathrm{~K}$ and self-field. In the cross-section, the reactions are observed to be nearly continuous along the interface. The plan view image shown in Figure $7 \mathrm{~b}$ was taken with the beam nominally parallel to the [001] direction in the film and substrate. The areas of the micrograph that exhibit a bright contrast are YBCO grains that are misaligned relative to the desired alignment of [001] YBCO $\|[001]$ of the ceria $\|[001]$ of the YSZ single crystal. It has been found that the density of misaligned grains increases as the severity of the interfacial reactions increases.

In the case of metal substrates and the optimal ceria thickness, the few areas of significant interfacial reaction that were found could be traced to the propagation of defects from the substrate. Such was the case for the samples in Figures $2 b$ and 4 , which had ceria thicknesses of approximately $90 \AA$ and a $J_{\mathrm{c}}$ values of $2.9 \mathrm{MA} / \mathrm{cm}^{2}$. The underlying reasons for the increased stability of the ceria buffer layer for certain optimal thicknesses is unclear at this point. Possibly an optimized stress state is obtained in the ceria buffer layer for a certain thickness as it bridges 


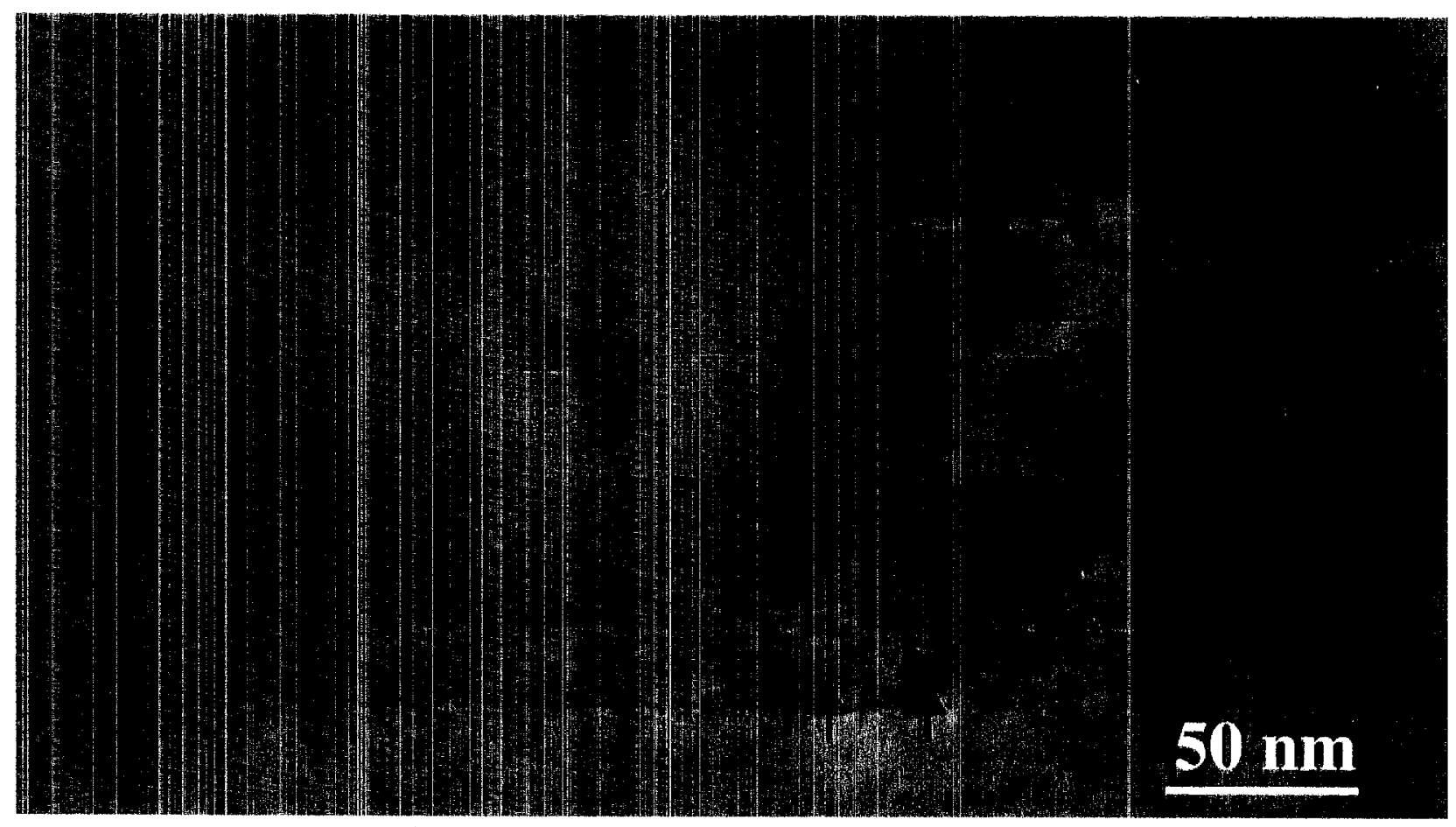

Higure 6: TEM micrograph of the interface of a $1 \mu \mathrm{m}$ thick YBCO film on a single-crystal YSZ substrate with an intervening $200 \AA$ thick ceria buffer layer. Only a few nano-sized interfacial reactions products could be found along the interface as indicated by the arrows above. The $\mathrm{J}_{\mathrm{c}}$ value of the film was $2.9 \mathrm{MA} / \mathrm{cm}^{2}$ at $75 \mathrm{~K}$ and self-field.

the lattice mismatch between the YSZ and the YBCO films. The polycrystalline nature of the IBAD template may explain the factor of two decrease in the optimal thickness of the ceria buffer layer when compared to the results on single crystal substrates. However, the overall results are clear. Signilicant enhancements in $\mathrm{J}_{\mathrm{c}}$ (up to $55 \%$ in the present work on IBAD YSZ templates) can be obtained in YBCO films when the thickness of the underlying buffer layer is optimized. In addition, many more defects in the YBCO films can be eliminated through a careful choice of substrate preparation conditions that reduce or eliminate substrate irregularities. Hence, one has the means to control and engineer the structure of a coated conductor in order to optimize its transport properties.

\section{CONCLUSIONS}

The roles that substrate defects and interfacial reactions play in the development of defects in YBCO films on IBAD YSZ templates and single-crystal YSZ substrates were examined. Substrate dofects can propagate through the intervening buffer layers and affect YBCO film growth. Hence, the preparation of the substrate surface prior to film deposition is a critical parameter in the fabrication of high-..Jc coated conductors. The thickness of the buffer layer immediately beneath the YBCO film can also be used to control the severity of the interfacial reactions. Optimal ceria thickness of $90 \AA$ and $200 \AA$ were found for the IBAD YSZ templates and YSZ single-crystal substrates, respectively. The use of the optimal ceria thickness significantly reduces the interfacial reactions and enhances the $J_{c}$ values of the YBCO films. In the case of TBAD YSZ, templates, the use of the optimal $90 \AA$ thickness resulted in a $1.5 \mu \mathrm{m}$ thick 

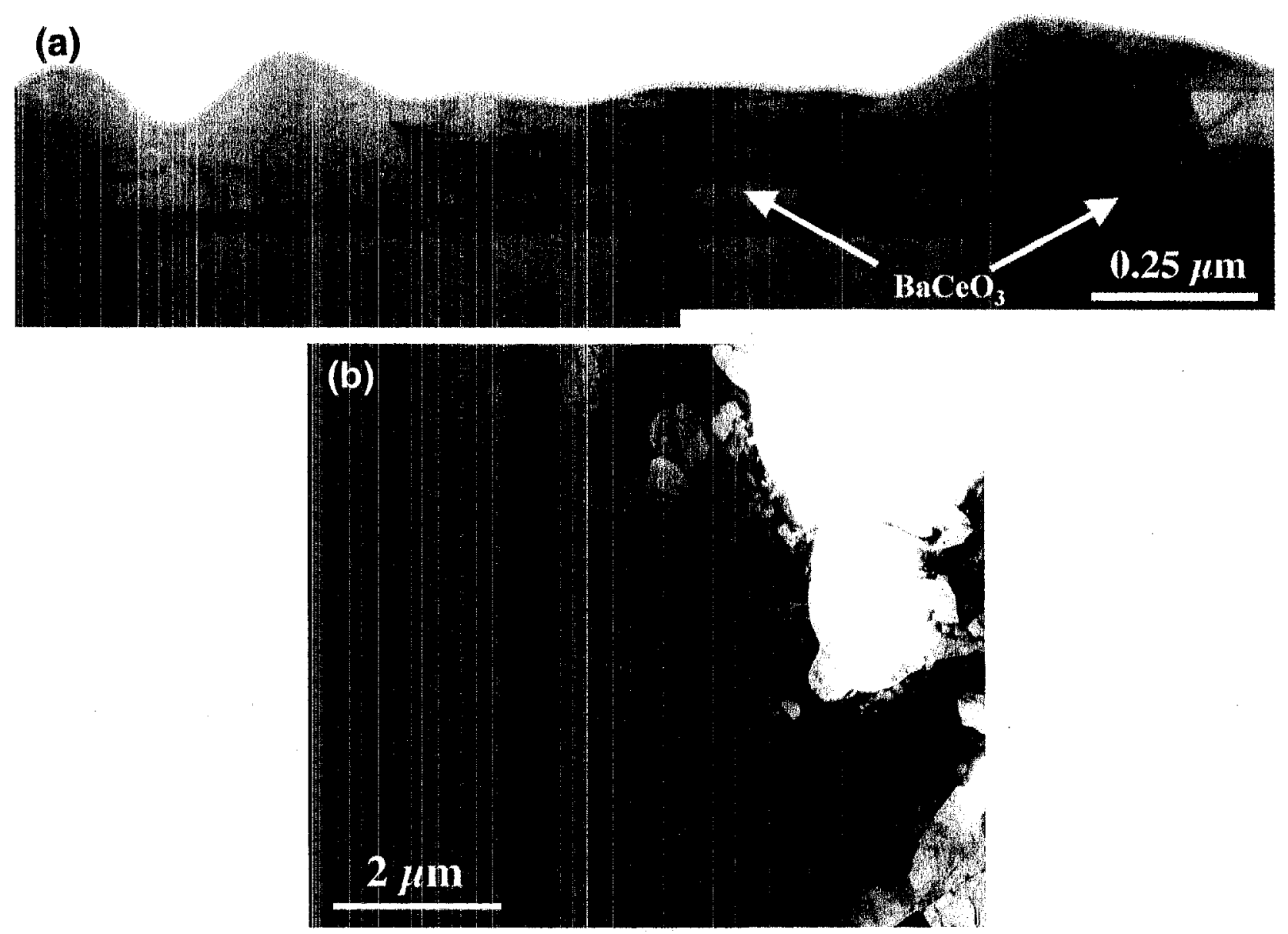

Figure 7: TEM images of samples with YBCO films on single-crystal YSZ substrates with intervening ceria buffer layer thickness of (a) $560 \AA$ and (b) $800 \AA$. The corresponding $\mathrm{J}_{\mathrm{c}}$ values were 0.8 and $0.35 \mathrm{MA} / \mathrm{cm}^{2}$. The cross-section in (a) shows a continuous interfacial reaction layer between the ceria and YBCO. The plan-view TEM image of (b) shows the misaligned YBCO grains in the film. The latter image was taken with the beam parallel to the [001] direction of sample. The areas of the sample that appear bright in the image are not aligned in this manner.

YBCO film having a $J_{s}$ level of $1.7 \mathrm{MA} / \mathrm{cm}^{2}$. Likewise, an optimal ceria thickness of $200 \AA$ on a single crystal YSZ substrate was used to produce a $1 \mu \mathrm{m}$ thick YBCO film with a $\mathrm{J}_{\mathrm{c}}$ level of 2.9 $\mathrm{MA} / \mathrm{cm}^{2}$. These results show that it is possible to control the development of the structure of the coated conductor during processing for enhanced $\mathrm{J}_{\mathrm{c}}$ values.

\section{ACKNOWLEDGMENTS}

This work was performed under the auspices of the United States Department of Energy, Office of Energy Efficiency and Renewable Energy, as part of a DOE program to develop electric power technology, under contract W-7405-ENG-36. 


\section{REFERENCES}

1. Y. Iijima, N Tanabe, O. Kohno, and Y. Ikeno, Applied Physics Letters 60 (6), 769-771 (1992).

2. R.P. Reade, P. Berdahl, R.E. Russo, and S.M. Garrison, Applied Physics Letters 61 (18), 2231-2233 (1992).

3. X.D. Wu, S.R. Foltyn, P. Arendt, J. Townsend, C. Adams, I.H. Campbell, P. Tiwari, Y. Coulter, and D.E. Peterson, Applied Physics Letters 65 (15), 1961-1963 (1994).

4. X.D. Wu, R.C. Dye, R.E. Muenchausen, S.R. Foltyn, M. Maley, A.D. Rollet, A.R. Garcia, and N.S. Nogar, Applied Physics Letters 58 (19), 2165-2167 (1991).

5. S.R. Foltyn, P. Tiwari, R.C. Dye, M.Q. Le, and X.D. Wu, Applied Physics Letters 63 (13), 1848-1850 (1993).

6. T.G. Holesinger, S.R. Foltyn, P.N. Arendt, H. Kung, Q.X. Jia, R.M. Dickerson, P.C. Dowden, R.F. DePaula, and J.Y. Coulter, Journal of Materials Research 15 (5), 222-240 (2000).

7. T.G. Holesinger, S.R. Foltyn, P.N. Arendt, Q. Jia, P.C. Dowden, R.F. DePaula, and J.R. Groves, IEEE Transactions on Applied Superconductivity 11 (1), 3359-3364 (2001).

8. M. Paranthaman, D.F. Lee, A. Goyal, E.D. Specht, P.M. Martin, X. Cui, J.E. Mathis, R. Feenstra, D.K. Christen, and D.M. Kroeger, Superconductor Science and Technology 12, 319-325 (1999).

9. M. Hasegawa, Y. Yoshida, M. Iwata, K. Ishizawa, Y. Takai, and I. Hirabayashi, Physica C 336, 295-299 (2000).

10. M. Gurvitch and A.T. Fiory, Applied Physics Letters 51 (13), 1027-1029 (1987).

11. C.T. Cheung and E. Ruckenstein, Journal of Materials Research 4 (1), 1-15 (1989).

12. G.L. Skofronick, A.H. Carim, S.R. Foltyn, and R.E. Muenchausen, Journal of Materials Research 8 (11), 2785-2798 (1993).

13. P. Merchant, R.D. Jacowitz, K. Tibbs, R.C. Taber, and S.S. Laderman, Applied Physics Letters 60 (6), 763-765 (1992).

14. P.N. Arendt, S.R. Foltyn, J.R. Groves, R.F. Depaula, P.C. Dowden, J.M. Roper, and J.Y. Coulter, Applied Superconductivity 4 (10-11), 429-434 (1996).

15. S.R. Foltyn, P.N. Arendt, P.C. Dowden, R.F. DePaula, J.R. Groves, J.Y. Coulter, Q. Jia, M.P. Maley, and D.E. Peterson, IEEE Transactions on Applied Superconductivity 9 (2), 1519-1522 (1999). 